\title{
APRENDER A EMPRENDER CON CÓDIGO ABIERTO
}

Mario Dehter ${ }^{1}$

\author{
${ }^{1}$ CEO de Es24 WebTV, producción de contenidos audiovisuales \\ $y$ programas de formación sobre innovación y emprendimiento \\ Profesor Extraordinario Visitante de la Facultad de Ingeniería de la Universidad FASTA \\ Correo electrónico: mariodehter@ufasta.edu.ar
}

Recibido: 15 de abril del 2015. Aprobado: 20 de julio del 2015.

Cómo citar este artículo: M. Dehter. "Aprender a emprender con código abierto". Ingeniería Solidaria, vol. 11, n. ${ }^{0} 18$, pp. 9-21, ene.-dic. 2015. doi: http://dx.doi.org/10.16925/in.v11i18.987

Resumen. En este artículo de reflexión, se analiza cómo la formación académica que se imparte en las facultades de ingeniería en la mayoría de las universidades iberoamericanas es monodisciplinar, es decir, especializada en alguna rama tecnológica, descuidando el aprendizaje de habilidades sociales que forman la base del talento para el liderazgo, los espíritus visionarios e innovadores y las actitudes y aptitudes que permiten hacer buenos negocios. Si bien educar para el emprendimiento es un tema instalado en toda la comunidad educativa a través de todos los niveles de enseñanza, esto no significa que se deba dejar de lado la reflexión académica entre enseñar a y aprender a emprender. Algunas propuestas son expuestas con el fin de mejorar la situación de la educación emprendedora en los programas de ingeniería y se sintetizan conceptos tales como open source learning, flipped classroom y entorno personal de aprendizaje (PLE). Se propone una renovación de estrategias didácticas para aplicar en las aulas de ingeniería los componentes del aprendizaje emprendedor con código abierto. Así, los docentes deben afrontar los riesgos implícitos en los desafíos con el fin de crear diseños instruccionales en los cuales los estudiantes aprendan y emprendan a través de sus propios intereses.

Palabras clave: aprender, competencias, didáctica, emprender, habilidades sociales. 


\title{
LEARNING TO LAUNCH ENTERPRISES With Open Source Code
}

\begin{abstract}
This article analyzes how academic training at the engineering faculties at most Iberian-American universities is monodisciplinary, in other words, specialized in a particular technological branch, while neglecting the learning of social abilities that are basic for leadership, visionary and innovative spirits and the attitudes and aptitudes that make for good businesses. While entrepreneurial training is a topic found throughout the educational community at all levels of teaching, this does not mean that academic thinking about the difference between teaching and learning to create an enterprise should be left out. The article includes proposals to improve the quality of entrepreneurial education in engineering programs and summarizes such concepts as open source learning, flipped classroom and personal learning environment (PLE). A renovation of didactic strategies is proposed for application in engineering classrooms of the components for learning to create an enterprise with open source code. Teachers must therefore deal with the risks implicit in such challenges in order to build instructional designs in which students learn and create enterprises through their own interests.
\end{abstract}

Keywords: learning, competences, didactic, enterprise, social abilities.

\section{APRENDER A EMPREENDER COM CÓDIGO ABERTO}

Resumo. Neste artigo de reflexão, analisa-se como a formação acadêmica que é ministrada nas faculdades de engenharia na maioria das universidades ibero-americanas é monodisciplinar, ou seja, especializada em algum ramo tecnológico, descuidando a aprendizagem de habilidades sociais que formam a base do talento para a liderança, para os espíritos visionários e inovadores e para as atitudes e habilidades que permitem fazer bons negócios. Embora educar para o empreendimento seja um tema instalado em toda a comunidade educativa em todos os níveis de ensino, isso não significa que se deva deixar de lado a reflexão acadêmica entre ensinar a e aprender a empreender. Algumas propostas são expostas com $o$ objetivo de melhorar a situação da educação empreendedora nos programas de engenharia e sintetizam-se conceitos tais como open source learning, flipped classroom e ambiente pessoal de aprendizagem (PLE). Propõe-se uma renovação de estratégias didáticas para aplicar nas aulas de engenharia os componentes da aprendizagem empreendedora com código aberto. Assim, os docentes devem enfrentar os riscos implícitos nos desafios com o objetivo de criar desenhos instrucionais nos quais os estudantes aprendam e empreendam por meio de seus próprios interesses.

Palavras-chave: aprender, competências, didática, empreender, habilidades sociais. 


\section{Introducción}

Es curioso que la pregunta ¿por qué fracasa la educación emprendedora en las universidades iberoamericanas? tenga un limitado conjunto, bastante predecible, de respuestas sin que nadie reformule la cuestión: ¿fracasa la educación emprendedora en las universidades iberoamericanas? Esto llevaría a analizar si todas las universidades iberoamericanas poseen programas de formación emprendedora para sus estudiantes y si, en caso afirmativo, se trata de programas transversales que abarcan el conjunto de todas sus carreras o a un segmento específico, como "a algunos estudiantes de economía y administración”; o si son, por otra parte, cursos optativos, cátedras abiertas o centros de asistencia para creadores de empresas, o componentes didácticos explícitamente incluidos en sus grillas curriculares.

\section{Que lo urgente no arruine lo importante}

De mis 30 años dedicados a la didáctica del emprendimiento, concluyo que no existe un acuerdo sobre qué significa educación emprendedora, y mucho menos de cuáles podrían ser los parámetros de evaluación que permitan establecer su grado de calidad $y$, en consecuencia, la efectividad de sus resultados.

Cada investigador y docente dedicado a la educación emprendedora tiene sus propios "relatos", los cuales giran en torno a cuestiones comunes: ecosistema socioproductivo, metodologías para la formulación de modelos de negocios y proyección de planes de inversión. En general, se orientan a aspectos más vinculados con los procesos de la administración de empresas que a la estimulación del espíritu emprendedor, porque se confunde la naturaleza humana del ser emprendedor con las capacidades profesionales del gestor de empresas.

Es como si la mayoría de los estudios y las recomendaciones de intervención de la educación emprendedora - sobre todo en las facultades de ingeniería - trataran de pasar balones de básquet a través de mangueras de riego: o revientan la pelota o destrozan la manguera, pero las semillas no germinan.

Aunque muchos estudiantes de ingeniería tengan la intención de convertirse en empresarios, no reciben ninguna formación útil dirigida a desarrollar su espíritu emprendedor. Todo queda librado a "su suerte", suponiendo que las competencias para formular proyectos de inversión y tener algunas nociones teóricas sobre la gestión comercial son las competencias necesarias que permitirían emprender la creación de negocios y un plan de vida con razonables expectativas de éxito.

En los últimos 15 años, con la pauperización de la economía global, la formación de emprendedores ha dejado de ser sólo una cuestión "políticamente correcta" para transformarse en una acuciante demanda social para que el sector educativo provea una "solución urgente".

En la urgencia se confunde la diferencia entre el emprendedor como el "talante fruto de la cultura" y el "talento integrado por competencias" para planificar y gestionar empresas. De esta manera, se asume que la pasión por emprender y la voluntad para hacerlo se pueden transferir mediante procesos normalizados de enseñanza-aprendizaje, tal como se enseña y se aprende, por ejemplo, cálculo de estructuras.

En el fondo del problema subyace una cuestión clave: todos hablamos de lo mismo, pero no estoy seguro de que todos asignemos el mismo significado a conceptos tales como "emprendedor" [1], "espíritu emprendedor", "competencia empresarial", "comportamiento fundador" y "cultura emprendedora".

Aunque muchos supongan que ya se ha ganado la batalla por instalar la "formación emprendedora" en la educación, los indicadores de la pobreza en Iberoamérica parecen indicar que estamos perdiendo la guerra. La educación emprendedora funciona mal, es lenta, tiene poco impacto, es costosa y derrocha muchos recursos. Si esto fuera una empresa, los accionistas reclamarían el cierre de esa línea de negocio para dedicar los recursos a algo más productivo.

No se trata sólo de cambiar de perspectiva para atacar el problema: también es necesario cambiar las armas y el conjunto de estrategias de la enseñanza del emprendedorismo y el aprendizaje del emprendimiento.

\section{Edificar desde arriba}

Es inútil plantarse en el extremo de la perspectiva económica, desde donde emprender es crear empresas siguiendo una oportunidad para lograr beneficios, o en las asunciones de los psicólogos conductistas que nos muestran un "ser emprendedor" como un conjunto de rasgos estereotipados 
de personalidad que se pueden replicar a través de procesos formativos lineales (dotación de información, sesiones de práctica, ejercicios prácticos) y estandarizados (aula, estudios de casos, exámenes de evaluación).

Si bien es posible enseñar a los estudiantes de ingeniería a ser innovadores y autosuficientes, para iniciar su propio negocio, no se están logrando resultados significativos porque el tema "educar para emprender" no está instalado en el corazón de la vocación de los docentes ni pertenece a la cultura institucional de las facultades de ingeniería.

Infortunadamente, la mayoría de los profesores universitarios de las facultades de ingeniería en Iberoamérica no son las personas idóneas para estimular y facilitar el desarrollo del espíritu emprendedor. Aunque poseen gran talento profesional en alguna especialidad tecnológica y destreza didáctica para transferirlo, utilizan diseños instruccionales que no resisten la turbulenta irracionalidad que puede entrar por una "ventana abierta" desde el "ruidoso patio emprendedor".

La naturaleza vertiginosa del ser emprendedor desafía el ordenamiento sistémico en la mayoría de las universidades, ya que generalmente los emprendedores actuamos sin tener todos los conocimientos ni los recursos de lo que intentamos hacer. Además, aunque con un discurso bastante creíble sobre articulación con el entorno social, en el ambiente académico subyace el mito según el cual el mundo de los negocios contamina la asepsia del claustro.

Esto está cambiando. No sólo por una renovación generacional del profesorado, la mayor participación de mujeres estudiantes y docentes, la presión social interna y externa sobre la renovación institucional de la universidad del siglo xxi, la voluntad política de facilitar la articulación del conocimiento académico con el entorno socioproductivo o la natural evolución de "las cosas". Sobre todo, se está evolucionando hacia el aprendizaje con código abierto porque los emprendedores están ganando terreno "en el patio", pese a lo que entorpece "su juego".

Los procesos normalizados en la enseñanza superior en Iberoamérica se resumen en una dinámica en la cual el docente ("quien da luz") imparte información a los alumnos ("quienes no están iluminados"), los cuales deben aprender a responder correctamente lo correcto para ser acreditados en porciones fragmentadas del conocimiento. La manera como el alumno integra la materia a las otras materias es su problema. Lo que el alumno hará en su futuro con lo aprendido en cada materia no es problema de cada docente. Más aún: la acreditación de la competencia para el ejercicio profesional del ingeniero se construye, a lo largo del trayecto formativo, sobre la certeza de las respuestas con que responde a sus profesores y no en la calidad de sus preguntas.

Sinceramente, no sé si hay un método mejor para aprender a construir edificios o diseñar motores. De hecho, no me sentiría seguro en la planta 26 de un edificio construido por un autodidacta. Pero en materia de educación emprendedora, es necesario abrir el código didáctico, invertir el rol del instructor que dota información y evalúa destrezas, por el de un facilitador de experiencias y, sobre todo, asumir que enseñar a emprender - con la metodología académica "al uso" y contenidos propios para la formación de administradores-es como pasar balones de básquet por dentro de mangueras de riego; simplemente aprender a emprender no funciona con la lógica académica.

Además, la revulsiva evolución de la cultura digital permite saltar muchas etapas del clásico proceso del emprendizaje: desde detectar una oportunidad, imaginar una idea, pasar por la formulación de un plan, hasta asumir una iniciativa para emprender. Ahora es posible avanzar de una manera muy rápida, a nivel global, encontrando un nuevo conjunto de problemas y desafíos, pero también de facilidades que permiten expandirse y acercar clientes, proveedores, consejeros, socios o empleados.

Aprender a emprender es en clave de código abierto porque no hay un patrón normalizado que se aplique para todos los estudiantes en todas las facultades, por todos los docentes y en cualquier tiempo y lugar.

En este sentido, debido a la creciente diversidad de la naturaleza del ser emprendedor, la formación emprendedora puede ayudar al estudiante de ingeniería emprendedor de tres maneras: (a) facilitando su conexión a redes internacionales de contactos y asesores, (b) preparándolo para las dificultades y oportunidades relacionadas con el trato a través de diferentes culturas, y (c) exponiéndolo a las diferentes maneras en que los negocios se hacen en todo el mundo.

Podemos evitar dar rienda suelta a emprender negocios con ideas imprudentes, a la vez que ayudamos a identificar y facilitar el aprovechamiento 
de los recursos innovadores que están presentes en todos los negocios con potencial de éxito. Esto significa la construcción de un entorno académico que incite a la creación de nuevas ideas y enfoques innovadores, con el fin de ser ingenieros felices y - si lo desean - hacer buenos negocios, más allá de la dotación de conocimientos encaminados a desarrollar las competencias que permitan resolver los problemas tecnológicos.

El lado oscuro de todo el problema de la educación para emprender radica en la incapacidad, por desconocimiento, de eliminar la actitud de emprender porque es cool, o evitar perder grandes talentos emprendedores porque muchos estudiantes no logran conectar su vocación con el futuro a largo plazo, en el cual deberán crearse un plan de vida en el que tenga cabida la ingeniería y su felicidad.

Estas cuestiones, así como el futuro y la felicidad de los estudiantes de ingeniería y los ingenieros, no se percibe como un problema de la facultad de ingeniería, cuya responsabilidad se limita a impartir la carrera de ingeniería y certificar las competencias aprendidas.

El corolario de todo esto es que las facultades de ingeniería deberían no sólo enseñar ingeniería a sus estudiantes para emprender. Deben comenzar a edificar por arriba, modelando el espíritu emprendedor de su profesorado, así como comenzar a educar a las empresas que recibirán el talento emprendedor de sus estudiantes que egresan como ingenieros.

La formación emprendedora eficaz es una solución de largo plazo que implica integrar la facultad de ingeniería con el ecosistema regional, con más puntos de anclaje que la mera formación de los ingenieros; esto es: (a) la estimulación emprendedora de sus docentes, (b) la auténtica formación emprendedora de sus estudiantes y (c) la articulación, integrada en su cadena de valor, del ecosistema regional con visión global.

\section{Estimular el talante y facilitar el talento emprendedor}

Los estímulos del contexto, para provocar su creación (cursos, concursos, subsidios, políticas promocionales...) no producen ningún efecto positivo de largo plazo por sí solos, sin que los potenciales emprendedores reconozcan en sí mismos su propia "identidad emprendedora" [2]. El proceso de emprender, aunque se inicie por cualquier circunstancia individual o social, siempre se basa en la intención personal de actuar como tal.

De esta manera, un punto de partida será ayudar a reconocer cuál es la propia naturaleza emprendedora para emprender, tanto a profesores como estudiantes, en un marco conceptual que implica:

1. La intervención del formador;

2. Los contenidos didácticos;

3. Las mejores metodologías; y

4. La estructura del programa de actividades para aprender a emprender.

Cuando se comienza a profundizar en las cuestiones que conciernen a los principios, los contenidos y las metodologías didácticas para la formación de emprendedores, se encuentra una disonancia cognitiva entre la teoría y la realidad.

Primero, los índices de empresarialidad (índice de cantidad de creación de nuevas empresas en relación con la población económicamente activa) en Iberoamérica no demuestran ninguna relación directa significativa entre los cursos para emprendedores y la creación de nuevas empresas. Segundo, una cuestión es la instrucción para la gestión empresarial, y otra la estimulación de la naturaleza emprendedora.

\subsection{Formar o facilitar}

Esta es la primera cuestión que debemos comprender: ¿estamos frente a la necesidad de formar emprendedores, o de facilitar a los emprendedores las experiencias de aprendizaje que les ayuden a sí mismos a consolidar su naturaleza emprendedora?

La educación para emprender como una intervención didáctica al uso - clases en la que se imparte información, se analizan algunos casos y se ejercitan algunas destrezas- está provocando el efecto contrario al esperado.

Se suelen presentar ejemplares "modelos de rol" que resultan ser lapidarios, porque desalientan el talante (espíritu), aunque se posea talento (conocimientos y habilidades); muchos estudiantes quedan atrapados en la duda: ¿seré capaz de ser tan genial y reunir tantos recursos como en este caso que me presenta el profesor?

Cuando se escribieron las bases acerca de cómo son los emprendedores, cómo se comportan, cómo 
aprenden, cómo viven, cómo piensan, por qué tienen éxito o por qué fracasan, existía un rango de causas muy vinculadas a las vocaciones profesionales, al talento innovador, a los intereses y a las aptitudes personales. Sin embargo, con la pauperización global de los últimos cuarenta años, especialmente en Iberoamérica, la mayoría de los emprendedores ya son gente que trata de huir del desempleo y la pobreza, construyendo nuevas alternativas para subsistir.

Hoy resulta erróneo responder a la pregunta ¿quiénes son emprendedores?, con las mismas conclusiones a las que arribaron investigadores en Estados Unidos o Inglaterra hace cincuenta años. Estos estudios describen procesos lineales de la evolución de la cultura emprendedora que, en realidad, con el tiempo van modificando todos sus factores, porque vivimos en un mundo finito y con limitaciones. Ningún sistema lineal funciona en un mundo finito.

\subsection{Pertinencia de la teoría con la práctica}

Esta es la segunda cuestión, más compleja que la anterior porque posee varias facetas, que debemos comprender: (a) ¿son pertinentes los clásicos conceptos teóricos con pretensión de validez universal, en los cuales se asientan el saber-saber, saber-hacer y saber-ser del emprendedor contemporáneo en el contexto específico de cada economía regional? y (b) ¿es pertinente seguir vinculando el emprendimiento con la vocación personal cuando desde las políticas públicas se está anclando al ser emprendedor con los problemas del desempleo y los paliativos para la pobreza?

Uno de los aspectos que demuestran los errores de asociar indiscriminadamente empresarialidad con desempleo es el de atribuir el alto índice de fracaso de las iniciativas emprendedoras a condiciones tales como el financiamiento insuficiente, la inexperiencia administrativa de los nóveles fundadores de empresas o su débil habilidad comercial.

Propongo un enfoque distinto de la clásica confusión entre "capacidad empresarial" y "cultura emprendedora"; porque, como he expuesto, cuando se espera lograr lo segundo formando para lo primero, el resultado es insatisfactorio.

No parece viable - tal como se está dando la moda de suponer- que la tasa de empresarialidad sea una variable dependiente del aumento de la tasa de desempleo, o de las inciertas oportunidades profesionales para los jóvenes egresados de las universidades. Vincular de forma dependiente la actividad emprendedora al desempleo no comprende las necesidades de las personas de crear, libremente, su propio modo y estilo de vida. Además, ¿qué calidad de emprendimientos se crean por el efecto del desempleo? ¿Sobrevivir es emprender?

\section{De formar emprendedores a facilitar el proceso de aprendizaje para aprender}

Las habilidades, las actitudes y los valores para emprender no dependen de la amenaza del desempleo, ni derivan de ninguna otra razón puramente económica.

Emprender es la acción de un sujeto independiente, de un equipo o de una organización con el fin de materializar una idea. Formar emprendedores es facilitar ese proceso.

El desarrollo emprendedor es el resultado de un proceso cognitivo que integra los conocimientos en las estrategias que cada uno pueda emplear para materializar sus iniciativas, contribuyendo con las cadenas de valor regionales y adoptando un estilo de pensamiento ordenado y replicable, el cual le permita resolver satisfactoriamente sus problemas.

\section{1 ¿Formadores o conectores?}

Se debe comenzar por considerar cómo la formidable evolución cultural y tecnológica contemporánea revitaliza conceptos tales como apofenia (conexiones que nunca existieron antes de ser creadas deliberadamente con el objetivo de crear un nuevo concepto, desarrollar un nuevo proceso o crear una nueva empresa) y serendipia (encuentros circunstanciales de conceptos o hechos que no se estaban buscando en forma deliberada), referidos a encuentros inusuales y relaciones entre destrezas y conocimientos aparentemente desarticulados. Por eso, ahora tiene más protagonismo el aprendizaje continuo e informal que la enseñanza enciclopédica. Son más importantes las nuevas vinculaciones entre los conceptos conocidos que los conocimientos en sí mismos; es más importante saber cómo aplicar el conocimiento que la capacidad de acumularlo. 
Esta evolución del diseño instruccional parece aparejar la incomodidad de los académicos al uso, en un mundo inestable en el que paradójicamente los temores al fracaso provocan un excesivo apego a la experiencia previa, al aprendizaje formal y a los hábitos. En general, aunque se intente explicar una y otra vez de muchas maneras que lo único constante es el cambio, todavía se le atribuye demasiada predominancia a los marcos de referencia que aquí propongo cuestionar, frente a la disrupción que provoca un mundo ubicuo.

Surgen nuevos espacios - físicos o virtuales- en los cuales la gente se informa, aprende, se divierte, trabaja o se relaciona con otras personas, de quienes no conoce ni el tono de sus voces, ni suele interesar su aspecto físico. Estos nuevos ámbitos de actuación personal y profesional precipitan el desarrollo de actitudes sociales y habilidades para comunicarse con nuevos códigos de intercambio. El nuevo paradigma es la sinergia (colaboración integrada de fuerzas). Por todos estos motivos, los emprendedores y sus emprendimientos necesitan conectores que colaboren, más que simple instrucción.

Así, propongo asumir que formar emprendedores es facilitar las condiciones propicias con el fin de madurar iniciativas emprendedoras, y esto no se reduce a una intervención didáctica por la que se imparten conocimientos para que el proceso cierre, afuera de un aula, en el desempeño de habilidades que permitan crear y gestionar empresas. Por eso, me refiero a aprender a emprender con código abierto: el aprendizaje proviene y cierra afuera del aula.

De esta manera, es posible discernir cómo el formador es, en realidad, un conector que hace posible la transferencia del aprendizaje teórico hacia la práctica en el mundo real, ayudando a establecer vinculaciones entre ideas, recursos, necesidades, soluciones, competencias profesionales, destrezas y actitudes. La clave de los programas para formar emprendedores es comenzar por animar la cultura emprendedora institucional en cada facultad de ingeniería, la vocación emprendedora de sus profesores y el espíritu emprendedor de sus estudiantes, todo con el aporte de los ingenieros egresados que hayan fundado sus propias empresas. Se trata de recrear una atmósfera propicia para articular vocaciones, oportunidades empresariales e iniciativas con el conocimiento tecnológico.

\subsubsection{El flujo de la innovación}

Cada vez se reconoce con más claridad que el capital intelectual eficazmente aprovechado a través de la innovación es el recurso más valioso para cualquier país y para el desarrollo de cualquiera de sus sectores. Ahora bien, en la educación tenemos que reconocer que las ideas originales no son suficientes. La innovación ya abarca todo el espectro cultural: desde la generación de una idea creativa hasta su comercialización; en especial, estamos comprobando que el éxito de la innovación depende de convertir los flujos del conocimiento en bienes y servicios que mejoren la calidad de vida de las personas.

Sin embargo, la innovación a la que se alude no se limita a ser una función del flujo de la tecnología o de nuevos materiales en los productos y servicios. Más bien, es el proceso de aprender a emprender y aplicar eficientemente las ideas eficaces. En un escenario caracterizado por organizaciones con límites más difusos entre sus respectivos sistemas internos de producción y de gestión, con las otras organizaciones dentro de sus respectivos ambientes regionales y extraterritoriales, los procesos de aprendizaje del emprendedor son ubicuos: carecen de límites en el tiempo, así como adentro de un lugar específico.

Es necesario atender menos al predominio del protagonismo o la autoría individual y reducir el énfasis en las capacidades autónomas para el trabajo personal, con el fin de destacar la importancia de la colaboración entre socios dinámicamente vinculados por proyectos.

El desafío del sistema educativo es revitalizar los valores emprendedores en una sociedad heterogénea, impactada positivamente por el formidable desarrollo tecnológico y económico amplificado por la popularización de las tecnologías de la información y comunicación (TIC), y la creciente diversidad cultural provocada por la internacionalización de las actividades socioeconómicas. Aunque también hay que tener en cuenta cómo - paradójicamente- estos mismos factores del desarrollo económico dificultan la realización personal de las jóvenes generaciones.

\subsubsection{El flujo de la educación}

Recomiendo que cuando se elabore una intervención didáctica, o todo un programa de formación para 
emprendedores, es necesario considerar estos tres factores:

a) Paradoja: más oportunidades, menos accesibles;

b) Paradigma: la cultura del empleo coexiste y se complementa con la cultura emprendedora; y

c) Barrera: la falta de empleo no significa falta de oxígeno ni de oportunidades.

Los contenidos de la educación para emprender tienen que facilitar la identificación de oportunidades y transformarlas en actividades económicas sustentables y personalmente gratificantes.

La batalla por modificar la educación orientada al desarrollo de competencias para el desempeño laboral todavía se está librando y no está ganada. Aunque se comienza a vislumbrar una incipiente educación emprendedora, aún domina la impronta de la creencia en que emprender es una opción de salida laboral para el estudiante; por ese motivo, los contenidos y las metodologías continúan siendo los mismos que los de la educación para el trabajo.

Preparar a las personas para emplearse en un mundo que ofrece menos empleos, pero sigue ofreciendo nuevas oportunidades, genera la desarticulación entre los currículos educativos y las expectativas sociales. El resultado de este salto de fase es la insatisfacción social por lo que provee el sistema educativo formal.

En la paradoja del desarrollo (cada vez más rápido surgen más oportunidades, pero cada vez son menos accesibles para la mayoría de las personas), la educación emprendedora en las carreras de ingeniería tiene que estar enfocada al largo plazo, ser transversal durante todo el trayecto de la carrera y desprenderse de la problemática del desempleo.

Las actividades didácticas cuyo fin es estimular comportamientos emprendedores cobran auténtica relevancia cuando logran inspirar iniciativas para emprender, que vinculen el aprovechamiento de oportunidades globales con las vocaciones profesionales y las cadenas de valor regionales.

Así, entonces, con el fin de enfocar, planificar y ejecutar intervenciones didácticas para la formación de ingenieros emprendedores, es necesario darle espacio al pensamiento no lineal, impedir que la lógica sea un límite del pensamiento, crear situaciones desconocidas que estimulen a los estudiantes a buscar nuevas soluciones a problemas cotidianos e imaginar cómo solucionar los problemas que vendrán.

No es una tarea fácil. Sin embargo, sí es posible. Requiere una institución que facilite los recursos, tolere y sepa gestionar la ubicuidad como sistema, y que los estudiantes participantes quieran aprender a emprender; no se trata simplemente de que se les enseñen algunas habilidades para sobrevivir.

\section{Aprender en clave de código abierto}

La reorientación desde la educación hacia el uso del aprendizaje emprendedor es la base estratégica para establecer los objetivos, diseñar los contenidos y las metodologías didácticas de los programas de estimulación de los comportamientos emprendedores. La formación emprendedora de los estudiantes de ingeniería tiene que enfocar sus iniciativas, y no la proyección supuesta sobre cómo deberán desempeñarse los ingenieros. Quizás, hoy son los estudiantes quienes tienen mucho más clara esta inversión del enfoque acerca de cómo estimular sus propios comportamientos emprendedores.

La pregunta ahora no es: $i s e$ pueden formar a los estudiantes de ingeniería para que sean emprendedores?; más bien, ahora cabe cuestionarse: ¿cuál es el mejor diseño didáctico de las intervenciones para que aprendan a emprender?

Hoy son necesarios nuevos componentes en los contenidos pedagógicos de los currículos académicos, con el fin de que los estudiantes de ingeniería maduren sus iniciativas emprendedoras a la vez que modelan sus competencias como ingenieros. Esto requiere reconvertir las clásicas metodologías didácticas con una renovada base conceptual, acerca de las experiencias de aprendizaje requeridas por las nuevas orientaciones estratégicas, territorialmente sensibles y que se puedan adaptar a diferentes perfiles individuales y a sus preferencias de estilos de aprendizaje.

\subsection{Renovar las orientaciones estratégicas}

En este marco conceptual que propongo, la estrategia de un programa para facilitar el aprendizaje emprendedor (diferente al concepto de formar emprendedores) en las carreras de ingeniería, tiene 
que tener estos tres pilares: (a) balance entre capacidades y actitudes, con un enfoque regional territorialmente sensible, (b) integración de las tecnologías digitales con el fin de crear ambientes de aprendizaje virtuales (ubicuos, interactivos y conectados con otros ámbitos socioeconómicos, culturales y tecnológicos extraterritoriales) y (c) revitalización de los modelos de rol cercanos al estudiante, tanto sobre casos de éxito como de fracaso.

Al referirme a nuevas premisas orientadas a estimular iniciativas y modelar comportamientos emprendedores, sugiero un cambio radical del enfoque didáctico: sobre todo en relación con la manera en que el sistema educativo formal califica a los estudiantes por la exactitud de las respuestas dadas a las preguntas que se les formulan para examinarlos, y no por el sentido crítico de las preguntas que ellos sean capaces de formular). No debemos estancarnos en un pensamiento tan simplificado como aquel que plantea que la educación forma empleados; más bien, podemos ver el problema de formar personas que en su mayoría saben responder muy bien, pero que tienen dificultades para elaborar sus propias preguntas. La formación emprendedora con código abierto para aprender a emprender tiene que tener nuevos estilos de preguntas, que requieren nuevos estilos de respuestas.

\section{2 ¿De qué estamos hablando?}

Debemos aclarar si estamos hablando de competencias emprendedoras, de cualidades del ser emprendedor, o de los comportamientos emprendedores; también será necesario aclarar si existe un orden jerárquico entre estos tres conceptos, tal como se da en el dilema “¿primero el huevo o la gallina?”. Personalmente, creo que sí: existe una escala de estadios que ayuda a identificar mejor cuáles son las necesidades de estimulación y formación que deben ser prioritarias según cada una de las fases:

1. Las cualidades emprendedoras son innatas. Su afloramiento depende del contexto o del ecosistema sociocultural de cada individuo y, en especial, de la actitud colectiva que cada comunidad en particular tiene hacia sus individuos más ingeniosos, creativos y proactivos. Poco y nada tienen que ver aquí los profesores, ya que se trata de una cuestión de la cultura impregnada por las intervenciones de todos los estamentos sociales de la comunidad a través de varias generaciones, y las cuales pueden o no estar favorecidas o entorpecidas por las interacciones entre diversas comunidades, comenzando por los padres de cada individuo en su edad temprana.

2. Los comportamientos emprendedores se aprenden. A lo largo de mi experiencia personal - como emprendedor y como especialista creando contenidos y metodologías didácticas para la formación de emprendedores- he aprendido que este aprendizaje de los comportamientos se logra mediante procesos de observación y práctica que podrían fundamentarse con la teoría de la autoeficacia de Albert Bandura [3].

3. Las competencias para emprender se estimulan y desarrollan mediante procesos de enseñanza-aprendizaje que pueden tener diferentes modelos de ejecución, tales como:

- El aprendizaje asistido en forma presencial o virtual impartido por un equipo de instructores-facilitadores, siguiendo un programa previamente organizado de actividades de estudio y prácticas.

- El autoaprendizaje durante el contacto directo y continuo con emprendedores en acción, o simplemente siguiendo uno o varios modelos de rol preferentemente cercanos (territorio o sector de actividad).

Entre la educación empresarial y la actuación de los emprendedores existe una gran brecha entre: (a) lo que se difunde en las investigaciones académicas, (b) el diseño de los programas educativos y (c) el espíritu empresarial en la práctica. Los programas educativos por lo general se fundamentan en conocimientos teóricos que describen conjuntos genéricos de cualidades empresariales, lo cual complica el diseño de la educación para emprendedores cuando no se consideran muy específicamente los rasgos dominantes de la cultura en la que se desenvuelven habitualmente sus destinatarios.

\section{3 ¿Cómo enseñar a emprender?}

El título de este apartado representa sólo un aspecto parcial de la cuestión, ya que en realidad la educación para el emprendimiento con código abierto comienza por aprender cuáles son los marcos teóricos que 
permiten establecer cuáles son los mejores contenidos y las metodologías didácticas para aprender a enseñar a aprender a emprender. No es un juego de palabras.

Con el fin de construir estrategias efectivas para la educación emprendedora en el ámbito universitario -incluso en las facultades de ingeniería en las que se podría asumir que es una cuestión superflua-, primero es necesario comprender las diferentes teorías del aprendizaje que se pueden aprovechar para el "desarrollo emprendedor".

Los especialistas en ciencias de la educación coinciden en que la educación sistémica debe estructurarse de manera tal que los conceptos complejos se desagreguen en componentes simplificados como secuencia de pasos que mejoran la coherencia del plan de estudios.

A finales del siglo $\mathrm{xx}$, se identificaron diferentes teorías del aprendizaje que subyacen a los programas específicos de educación emprendedora, tales como las teorías del aprendizaje generativo [4], el aprender haciendo [5] y la taxonomía del aprendizaje cognitivo por objetivos [6].

Cada una de estas teorías del aprendizaje implica conceptos fundamentales para comprender y facilitar el aprendizaje de las cualidades emprendedoras que deben enseñarse desde una edad temprana, así como la manera de ir escalonando prácticas didácticas a medida que las personas van madurando y transitando por sus respectivos niveles de instrucción.

Sin lugar a dudas, es posible orientar todo el proceso educativo que se imparte desde las escuelas infantiles hasta los cursos de posgrado, como un proceso integrado por componentes simplificados que van consolidando actitudes y cualidades del comportamiento emprendedor.

\subsubsection{La teoría de aprendizaje generativo}

Se basa en la suposición de que el cerebro construye relaciones significativas entre los conceptos desconocidos, la experiencia adquirida y los conocimientos conocidos relevantes. El aprendizaje es enfocado como un proceso generativo por el que las personas construimos significados a partir de nuestros propios recuerdos, el conocimiento que adquirimos y la experiencia lograda.

\subsubsection{La teoría de la experiencia basada en el aprendizaje, o aprender haciendo}

Propone que los estudiantes aprendan mediante su implicación directa en experimentaciones y ejercicios, en los cuales deben tomar decisiones y asumir personalmente las consecuencias de esas decisiones. Se trata de provocar la participación activa de los estudiantes en situaciones de resolución de problemas de la vida real con significado personal.

\subsubsection{La taxonomía del aprendizaje cognitivo por objetivos}

Propone un marco teórico para la clasificación de objetivos cognitivos en la educación. Se trata de una disposición jerárquica de los siguientes objetivos cognitivo-conductuales:
1. Conocimiento
2. Comprensión
3. Aplicación
4. Análisis
5. Síntesis
6. Evaluación

De acuerdo con esta jerarquía, el objetivo fundamental de la educación es mejorar las capacidades de pensamiento.

\subsection{Ahora la curiosidad resulta una habilidad tan valorada como la memoria}

Si profundizamos en los tres enfoques teóricos de los procesos formativos, descubrimos los hilos conectores que nos ayudarán a comprender que, en la práctica, la educación reglada generalmente se ha centrado en torno a las categorías más básicas de la instrucción de conocimientos, los esfuerzos para comprender y recordar conceptos.

Hace muy pocos años se comenzó a percibir un alentador cambio hacia la predominancia de la práctica y el aprendizaje, en el cual las preguntas que formulan los estudiantes comienzan a tener una relevancia tan significativa como las respuestas que les exigen sus profesores. 
Ahora, falta otro paso para aprovechar la comprensión de cómo la educación emprendedora se entronca con conexiones entre varios marcos teóricos y no en un enfoque excluyente de la educación académica: reconocer que el desarrollo de los comportamientos emprendedores no es el resultado de una secuencia de instrucciones para desarrollar determinadas competencias (planificar, administrar, comunicar, negociar), sino que se trata de un proceso de aprendizaje que vincula ideas, contextos socioculturales y oportunidades.

El problema es la confusión imperante en la educación al uso entre lo que son los comportamientos emprendedores y las capacidades empresariales, combinada con la atroz reducción según la cual el talento emprendedor se trata de una mera competencia para crear o gestionar empresas.

La abrumadora mayoría de los educadores considera que la educación emprendedora no le concierne a la facultad de ingeniería; pese a esta cuestión, es necesario reconocer que todos los educadores - en todos los niveles- están preocupados por estimular la curiosidad de sus estudiantes, alentar su capacidad creativa y facilitarles el desarrollo de sus habilidades para tomar decisiones y resolver problemas.

De tal manera que muchos creen que lo que se llama educación emprendedora se trata de una cuestión meramente formal sobre cómo denominamos la educación; no perciben su gran diferencia semántica, porque entre la educación emprendedora y la educación perciben la misma intensión de estimular la curiosidad, la capacidad creativa, las habilidades para tomar decisiones y resolver problemas.

Quizás ahora se comprenda mejor que antes de preguntar: “icuáles son los motivos por los que fracasa la educación emprendedora?”, habrá que responder a la pregunta "ifracasa la educación emprendedora?" Como en el caso de "aprender a emprender" y "aprender a enseñar a emprender a emprender": no es un juego de palabras.

Si la educación emprendedora no existe en las facultades de ingeniería, no puede fracasar. Estaremos hablando, en el mejor de los casos, de contenidos didácticos y diseños instruccionales dirigidos a ingenieros capacitados para planificar y administrar empresas, pero no sobre emprendedores con iniciativas para crearlas.

\section{Componentes del aprendizaje emprendedor con código abierto}

Naturalmente que las intervenciones cuyo fin es facilitar el aprendizaje emprendedor en el ámbito de las facultades de ingeniería (programas, seminarios, cursos o talleres) implican contenidos didácticos necesarios para el desarrollo de las competencias que permiten planificar y gestionar empresas, diseñar y controlar procesos tecnológicos y administrativos, negociar, detectar oportunidades, analizar y vincularse con el mercado, etc., pero también hay que saber cuándo conviene vender o transformar el emprendimiento.

Todo esto incluye conocimientos sobre gestión administrativa, dominio de la tecnología vinculada con la naturaleza del emprendimiento, marketing, regulaciones legales que facilitan o entorpecen el emprendimiento, dominio del idioma inglés (por lo menos un nivel que permita entender un texto y dialogar con un extranjero no hispanoparlante), y lo que considero como cuestiones más críticas: (a) cómo conseguir y gestionar con eficacia los recursos necesarios para ponerse en marcha y sostener el emprendimiento a largo plazo; (b) cómo y cuándo crecer invirtiendo en el desarrollo organizacional y escalando la complejidad de los procesos de producción o comercialización; y (c) cómo reclutar, retener y gestionar un equipo de trabajo con colaboradores de gran talento (internos y externos), los cuales complementen las debilidades y apuntalen las fortalezas personales del ingeniero emprendedor y de su emprendimiento.

En este punto, sugiero focalizar los componentes didácticos que cobran gran relevancia en el marco que hemos estado tratando hasta aquí, y en relación con la tendencia que sigue el mundo contemporáneo a partir de las disrupciones que implican: (a) la crisis financiera global iniciada a mediados de la década del 2000; (b) el creciente impacto de la deslocalización territorial de los procesos de producción y comercialización; y (c) lo que todavía no sabemos que puede seguir ocurriendo como consecuencia de las disrupciones (a) y (b).

\subsection{Habilidades sociales para emprender}

Con el objetivo de abandonar la orientación lineal de intervenir hacia el emprendedor y adoptar la 
perspectiva desde la comunidad emprendedora, para facilitar el aprendizaje emprendedor, es necesario incorporar en los procesos componentes didácticos que: (a) ejerciten la habilidad para construir y gestionar redes de relaciones sociales personales y profesionales de apoyo, para apalancar el desarrollo de ideas y la puesta en marcha de las iniciativas que integren múltiples actores públicos, privados, extraterritoriales y multidisciplinarios; (b) fortalezcan las habilidades sociales que den valor a los conocimientos tecnológicos y las competencias para planificar y gestionar negocios (por ejemplo, el optimismo, el buen humor y la curiosidad); (c) impregnen el saber tecnológico y la habilidad empresarial con dimensiones sociales como:

- Qué sabe y qué quiere la gente.

- Qué es lo que la gente considera tecnología fácil y atractiva.

- La tendencia de las modas y las necesidades que aún no existen.

\subsection{Audacia social para emprender}

Confío que haya quedado claro cómo las habilidades sociales para interactuar con otros juegan un papel fundamental para el éxito profesional y financiero. Simple. ¿Por qué?

Cuando se observa cómo actúan las personas en sus respectivos ámbitos de trabajo, o en sus entornos familiares, jugando fútbol o en misa, se perciben diferencias significativas en todas las dimensiones de sus personalidades: desde su aspecto físico hasta su conciencia sobre lo que ocurre en su entorno, su capacidad para vincularse adaptativamente con las otras personas según las circunstancias que les toca vivir, su grado de extroversión, etc.

Considero que, para el buen desempeño de los comportamientos emprendedores eficaces, la "adaptabilidad" (capacidad para relacionarse eficazmente con diferentes tipos de personas en diferentes circunstancias) es, aún más que la "empatía" (capacidad para comprender las situaciones desde la perspectiva de los interlocutores), la más fundamental de todas las habilidades sociales.

De los casos próximos que conozco, los empresarios con personalidades mejor "adaptables" son quienes han logrado mayor apoyo financiero de terceras personas para sus empresas. No es una casualidad.
Aunque nunca he podido encontrar una investigación científica que demuestre la relación entre la "adaptabilidad social" y la "obtención de capital de riesgo", entiendo que la vinculación se basa en que las personalidades más adaptables son más capaces de crear mejores relaciones sociales con las personas apropiadas, en los lugares correctos, en los momentos oportunos.

El grado de eficacia de la adaptabilidad varía mucho en función de la edad, las experiencias personales y profesionales previas, y la capacitación que permita desarrollarla y ejercitarla. En estos términos, insisto, resulta muy beneficioso que los programas formativos para emprendedores ayuden a desarrollar un alto nivel de adaptabilidad social.

Aquí entran en juego otros factores del comportamiento social, como la "audacia" o la capacidad para relacionarse con personas extrañas.

Se ha preferido proponer este componente didáctico en forma separada de las habilidades sociales (descritas en el apartado anterior), ya que merece ser un tema transversal en toda la formación de grado universitario, aunque generalmente se le trata como un aspecto secundario cuando se enfocan problemas de evaluación de riesgos.

Suelo sugerir a mis estudiantes que reflexionen acerca de estas cuestiones: (a) ¿estás a gusto con todas las personas, sean jóvenes o viejos, hombres o mujeres, de diferentes creencias religiosas, nacionalidades o afiliación política?; (b) ¿puedes hablar sobre cualquier tema con cualquier persona?; y (c) ¿tienes algún problema para presentarte tú mismo ante una persona desconocida?

En general, las respuestas facilitan comprender la relevancia de la habilidad para adaptarse socialmente y el grado de osadía social, tanto para comenzar a ejercer una profesión independiente, como durante el proceso de start-up de nuevos emprendimientos, la gestión de innovaciones o reorganizaciones empresariales.

De manera tal que, si bien las habilidades sociales más útiles para los empresarios pueden variar en función de la fase de desarrollo de sus empresas, hay que considerar que es necesaria una cuota razonable de audacia para:

1. Entablar nuevas relaciones interpersonales en diferentes contextos, con diferentes estilos de personalidades. 
2. Asumir algunos riesgos (mesurados y ponderados), con el fin de alcanzar metas financieras y una adecuada dimensión empresarial con el plan de vida que se desea emprender.

\subsection{Construcción y gestión de redes de relaciones sociales personales $y$ profesionales de apoyo (networking)}

No me gusta (ni sugiero) aferrarse a las ideas que parecen indiscutibles, pero el tiempo demuestra que sólo en un contexto favorable combinado con un ambiente empresario dinámico es posible promover el surgimiento y la formación de emprendedores.

Pero, ¿qué es un contexto emprendedor favorable? De manera muy resumida, se trata de un ambiente sociocultural (normas, usos, costumbres, creencias, valores, actividades económicas, cadenas de valor, tecnología y recursos disponibles), capaz de vincular las preocupaciones y necesidades sociales con los intereses y las soluciones empresariales, y en el cual predomina la preocupación compartida por un armonioso co-funcionamiento entre la sociedad y sus empresas.

En principio, esto implica compartir el conocimiento entre todos los sectores y niveles dela sociedad y, por ello, son necesarias redes vinculantes. Cuanto mayor y mejor sea la cantidad de vínculos, la frecuencia y la calidad de las interacciones interpersonales, mejor será el contexto propicio para aprender a emprender y el desempeño de los emprendedores. Algo básico es que cualquier proyecto emprendedor necesita intenso capital social local, regional y extraterritorial, con el fin de ser creado, puesto en marcha y desarrollado con razonables expectativas de éxito.

\section{Conclusión}

En palabras del director de orquesta Riccardo Muti, Premio "Músico del Año 2001" conferido por la Sociedad América Musical, "estamos siempre en la mitad del camino y quizás nunca podamos llegar al otro lado del río porque detrás de cada nota habita el infinito..." [7].

Vale la pena que los profesores de ingeniería corran el riesgo de convertirse en animadores de la cultura emprendedora en sus respectivas facultades y en facilitadores del aprendizaje emprendedor de sus propios estudiantes. Aunque su especialidad sea el diseño de antenas ópticas de tamaño nanométrico dirigidas a realzar la emisión espontánea de luz procedente de puntos cuánticos semiconductores [8], asuma que usted puede ser un excelente facilitador de aprendizaje emprendedor para sus estudiantes en su facultad de ingeniería. A veces hay que emprender sin saber todo lo necesario, y se asumen varios riesgos hasta comiendo una aceituna.

\section{Referencias}

[1] M. Dehter, Ideología emprendedora. Impacto de las reglas del pasado en el juego del presente. [En línea]. Disponible en: http://www.mariodehter.com/ publi/ ideologia_emprendedora.pdf. Último acceso: mayo 22, 2015.

[2] M. Dehter, Factores para el surgimiento emprendedor. Enfoque de las identidades, la intencionalidad y la actuación emprendedora. [En línea]. Disponible en: http://www.mariodehter.com/publi/factores_emprendedores.pdf. Último acceso: mayo 22, 2015.

[3] A. Velásquez Fernández, Revisión histórico-conceptual del concepto de Autoeficacia. [En línea]. Disponible en: http://bit.ly/autoeficacia2012. Último acceso: mayo 22, 2015.

[4] A. Ontoria, J. P. R. Gómez y A. Molina, Potenciar la capacidad de aprender y pensar: modelos mentales $y$ técnicas de aprendizaje-enseñanza. Madrid, España, Ed. Narcea, 1999.

[5] Sujeto, educación y sociedad, John Dewey y la Escuela Pragmática. [En línea]. Disponible en: http://sujetoeducacionysociedad.wordpress.com/2011/11/22/ john-dewey-y-la-escuela-pragmatica. Último acceso: mayo 22, 2015.

[6] C. Perez y M. F. Sepúlveda, Taxonomía de Benjamín Bloom. [En línea]. Disponible en: http://mafrita. wordpress.com. Último acceso: mayo 22, 2015.

[7] R. Muti, El arte de la dirección musical [video]. Disponible en: http://youtu.be/xhZct1H4bxI. Último acceso: mayo 22, 2015.

[8] Noticias de la Ciencia y la Tecnología, Antena óptica para amplificar la luz espontánea de átomos, moléculas y puntos cuánticos. [En línea]. Disponible en: http:// noticiasdelaciencia.com/not/12603/antena-optica-para-amplificar-la-luz-espontanea-de-atomos-moleculas-y-puntos-cuanticos/. Último acceso: mayo 22, 2015. 